\title{
Design and Implementation of Stream Cipher Using Neural Network
}

\section{Siddeq Y. Ameen}

Dept. of Computer Eng. and I.T.

University of Technology/Baghdad

Safwan Omar Hasoon

Dr.safwan1971@yahoo.com

College of Computers Sciences

and mathematics

University of Mosul

\author{
Mazin Z.Othman \\ Technical College of Mosul \\ Commission of Technical Education \\ Moyed Abud Al-Razaq \\ Iraqi Committee for Computer \\ and Informatics
}

Received on:24/11/2008

\section{ABSTRACT}

The centaral problem in stream cipher cryptograph is the the difficulty to generate a long unpredicatable sequence of binary signals from short and random key. Unpredicatable sequence are desirable in cryptography because it is impossible, given a reasonable segment of its signals and computer resources, to find out more about them. Pseudorandom bit generators have been widely used to construct these sequences.

The paper presents a PN sequence generator that uses neural network. Computer simulation tests have been carried out to check the randomness of the generated through statistical tests. There tests have shown the successful PN sequence generator passes all the recommended tests. The paper also proposes and validates the data encryption and decryption process using neural network instead of using traditional methods (Exclusive or). This task increases the difficulty in the breaking the cipher.

Keywords: Neural Network, Cryptography, Stream Cipher

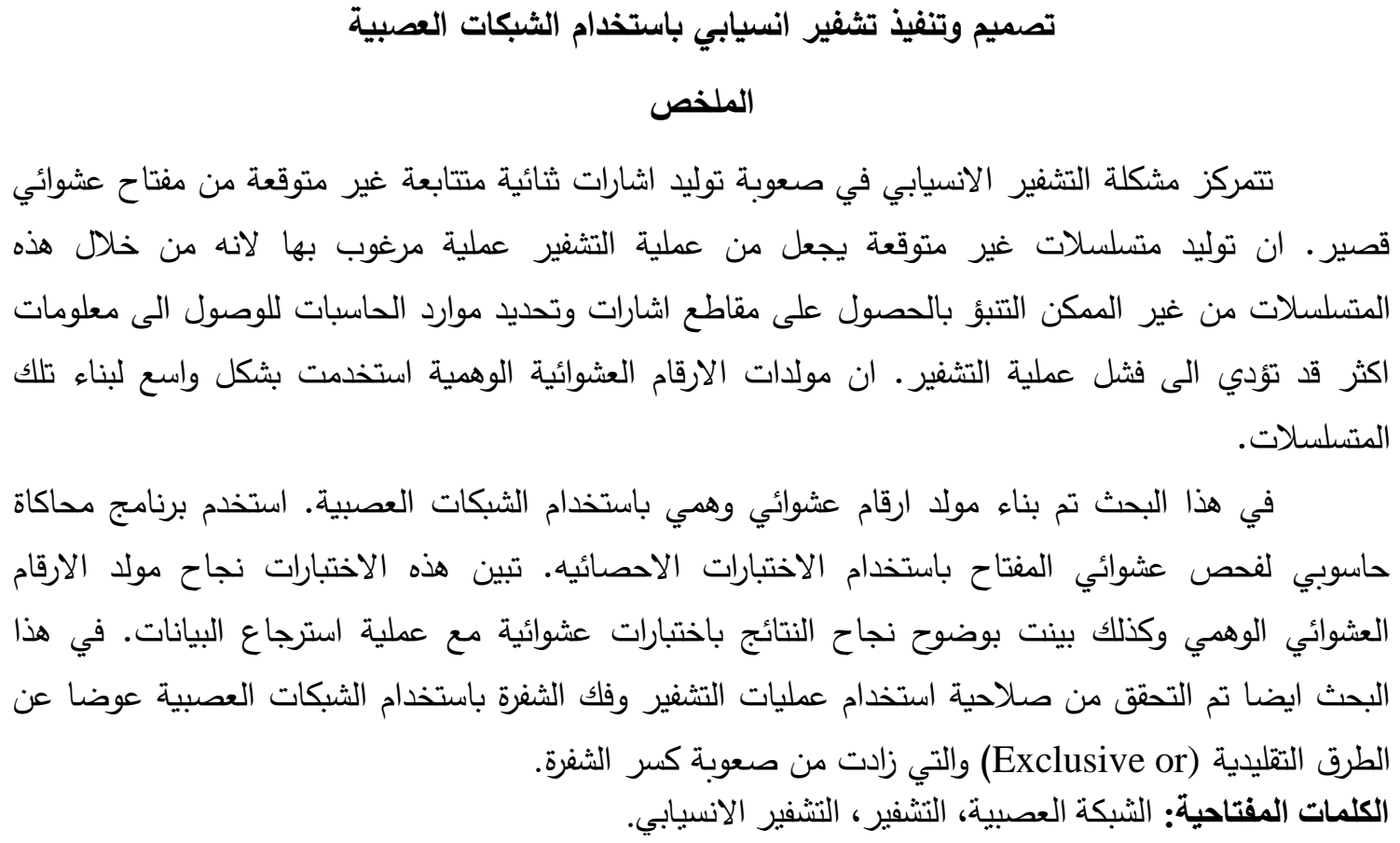




\section{Introduction}

Stream ciphers can be designed to be exceptionally fast, much faster than any block cipher. They are used in applications where plaintext comes in quantities of unknowable length, for example, a secure wireless connection (e.g. W-LAN and Bluetooth). If a block cipher were to be used in this type of application, considerable bandwidth would end up being wasted by padding, since block ciphers cannot work on blocks shorter than their block size. For example, if a 64-bit block cipher received separate 32-bit bursts of plaintext, half of the data transmitted would be padding. Stream ciphers eliminate this by operating on the smallest unit that can be transmitted[1].

Further advantages of stream cipher systems are:

1- Speed of encryption/decryption: since each bit of the message is encrypted independently of other plaintext bits, each bit can be encrypted as soon as it is read.

2- Low propagation error: since each bit is encrypted and decrypted separately, decryption of a bit that have been corrupted in transmission produces an error to the one corresponding message bit and no others.

An Artificial Neural Network (ANN) is an information processing paradigm that is inspired by the way biological nervous systems, such as the brain. The key element of this paradigm is the novel structure of the information processing system. It is composed of a large number of highly interconnected processing elements (neurones) working in unison to solve specific problems. ANNs, like people, learn by example. An ANN is configured for a specific application, such as pattern recognition or data classification, through a learning process. Learning in biological systems involves adjustments to the synaptic connections that exist between the neurones[3]. Researchers have also tried to use neural networks in Cryptography. In January 2002, the Physicists Kanter, Kinzel and Kanter proposeda new key exchange protocol between two parties $A$ and $B$. It uses the new notion of chaotic synchronization, which makes it possible for two weakly interacting chaotic systems to converge even though each one of them (viewed individually)continues to move in a chaotic way[4]. Neural network now typically have size of hundreds input and output allowing a huge mapping function to be implemented. Also the non-linearity produiced by neural network mapping is desired in cryptography. The implementation of neural networkcan be reduced to complexity of one using existing hardwre technologies[5].

\section{Stream Cipher}

Most stream ciphers consist of a pseudorandom number generator (PRNG).For encryption the PRNG is initialized with a key and provides its output as a sequence of bits known as the (pseudo) random key stream. Randomness is very important because it completely destroys any statistically properties in the message. Key stream generators are often constructed using linear feedback shift registers (LFSR). The LFSR method is an attempt to simulate a one-time pad by generating a long key sequence from a little information. As with any such attempt, if the key is shorter than the message itself, breaking part of the ciphertext gives the cryptanalyst information about other parts of the ciphertext. For an LFSR, a known plaintext attack can reveal parts of the key sequence[1].

The key stream is combined (XOR) with the plaintext bit by bit typically by an XOR gate. To decrypt the message the ciphertext bits are XORed with the corresponding key stream bits again. So an error in a single bit of ciphertext results in a single bit of plaintext error. This is very useful when the transmission error rate is high[6]. 
Some ciphers, called self-synchronizing stream ciphers, use several previous ciphertext bits to compute the key stream. They depend on the data and its encryption. A single-bit error will result in a long burst of garble, but the cipher will eventually recover from a lost bit after the damaged and incorrect bit falls off the shift register. The opposite are the synchronous stream ciphers, in which the key stream is generated independently of the plaintext and the ciphertext. They generate bits from a source other than the message itself. The simplest such cipher extracts bits from a register to use as the key. The contents of the register change on the basis of the current contents of the register. Most stream cipher designs are for synchronous stream ciphers[1].

The one-time pad is a form of stream cipher that can be proven secure. In this cipher each key character is randomly generated and is used only once[7]. The ciphertext gives opponent no additional information on the plaintext[8].

\section{Artificial Neural Networks}

Artificial neural networks (ANNs) are highly parallel interconnections of simple processing elements or neurons that function as a collective system. There exist various problems in pattern recognition that humans seem more efficient in solving as compared to computers. Neural networks may be seen as an attempt to emulate such human performance. These networks can be broadly categorized as those that learn adaptively by updating their connection weights during training and whose parameters are timeinvariant.

Artificial neural network consists of a large number of simple processing elements (PEs) densely interconnected, analogous to neurons of human brain. The neural network is made up of several layers of processing elements connected together via unidirectional signal channels associated with weights, analogous to synapses. These processing elements work in unision to slove a problem. The knowledge of the networks represented by its weights[9]. Every useful artificial neural net has a minimum of three layers: an input layer through which data is given to the network, an output layer that holds the response relative to the input and optional layer between the input and output layers called the hidden layer where learning takes place. The number of neurons in the input and output layers can be determined by the number of input and output variables in the physical system. The number of hidden layers and the nunber of neurons in the hidden layers are arbitrary and can vary from zero to any finite number.

Figure(1) shows a simple network architecture with four inputs and one output.

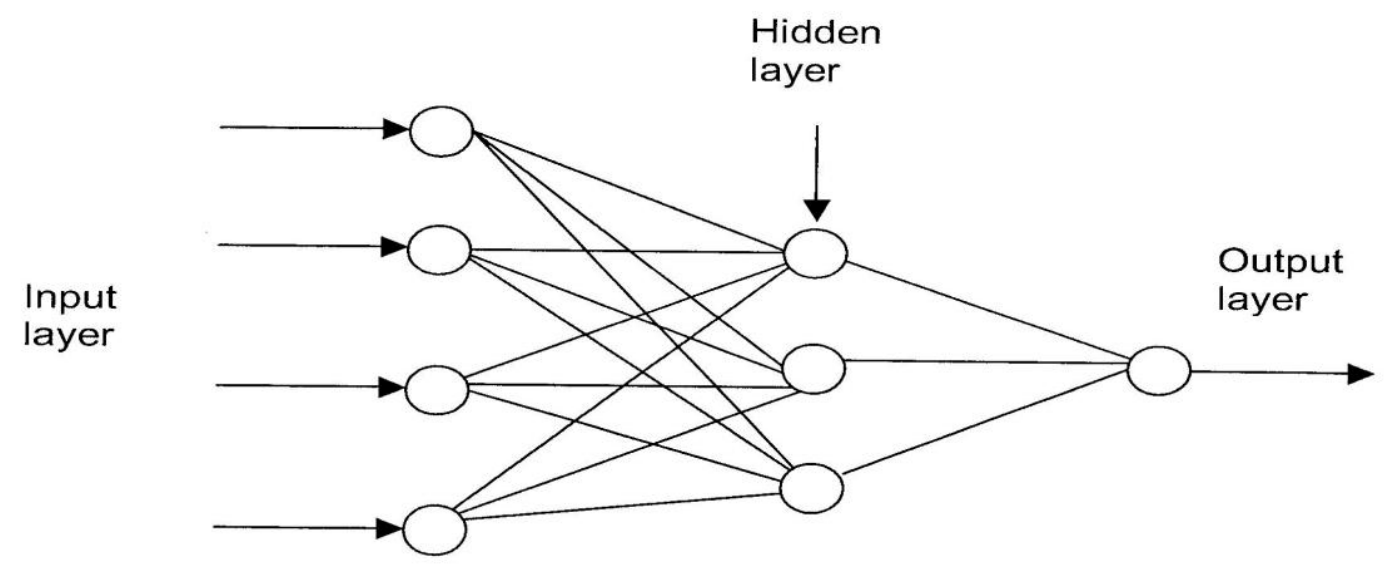


Figure.(1):A simple neural network architecture

With the given inputs and the related outputs, the network learns how the inputs of each data set are associated to the output. The network then continuously refines and organizes itself by adjusting the synaptic weights to fit the data, so that it produces relatively accurate response for a given input. Information processing of a single processing element is shown in Figure(2) Multiplying the input $\mathrm{X} i$ by weight $\mathrm{W} i$ can approximate the effect of a particular input unit. These weighted signals are added up to produce overall internal activation for the processing element. This activation level is passed through a transfer function, which produces an effective signal 'a'. If the activation is beyond a certain threshold, then the system gives the output response ' $y$ '.In Figure(2) there are $\mathrm{n}$ inputs with signals $X_{1}, X_{2}, \ldots, X_{n}$ and corresponding weights $W_{1}$, $W_{2}, \ldots, W_{n}$.

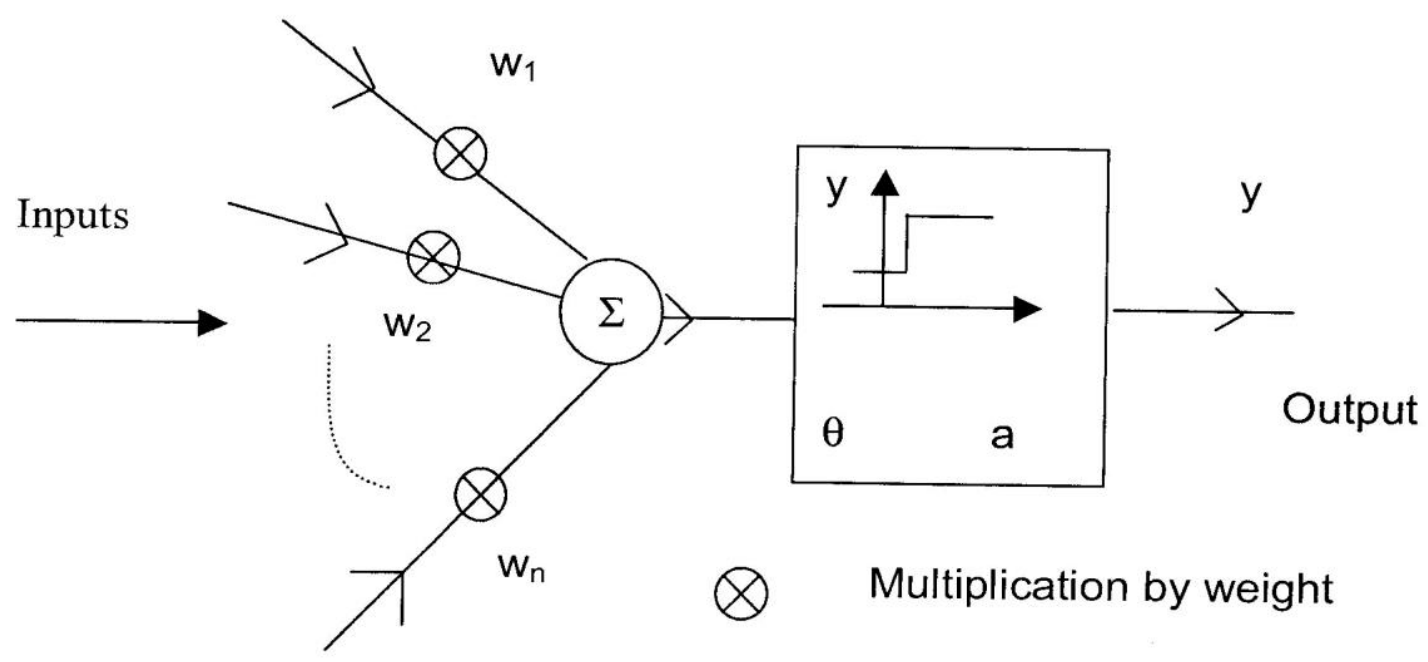

Figure.(2):Information processing of a single process element

The signals shown are Boolean value with values 'l' or '0' only. The system activation $\mathrm{a}$ is given by [10],

$$
a=w_{1} x_{1}+w_{2} x_{2}+\ldots+w_{n} x_{n}
$$

With this system activition, the output $y$ is given by,

$$
\begin{array}{ccc}
y=0 & \text { if } & a \leq \theta \\
y=1 & \text { if } & a \geq \theta
\end{array}
$$

where $\theta$ is the threshold that define the output. Generally there are two types of neural network archticecturte, Feed-forward networks and the Feedback networks.

In neural network, there are several network topologies. Each topology is designed to solve a specific problem or to represent certain point of view. Each topology has some drawbacks and benefits. These topologies can be categorized in three main categories: 
fully interconnected, feed forward connection, and mixed connectivity[11,12].

In Feed forward connection, the neurons are organized in multiple layers, so that a neuron in a certain layer is connected only to neurons on the next layer as shown in Figure (2). Multilayer perceptrons belong to this category [9].

\section{The Proposed Artificial Neural Network PN Sequence Generator}

The generation process of the key using backpropagation neural network cosist of there phases. The first phase is the feedforward and the second is a backpropagation process if there are errors. The third phase concerned with weights adjustments.

During a feedforward where input data is transmited from input unit $P_{i}$ and calculate the activition function and sends its input data to each hidden unit $h_{i}$ in hidden layer. In each hidden unit, the output of activition function is calculate to transform into output unit.

During training phase each output unit is compared with the target to determine the error rate. When the error is found, the error is propagate back to the input layer for weights adjusting(third phase). This process is repeated until the desired output obtained.

The algorithm below shows the procedure to achives the three phases:

1.Initiliazation network weight values

2.Sums weighted input and apply activition function to compute the output of the hidden layer using:

$$
h_{i}=f\left(\sum_{i=1}^{n} P_{i} W_{i j}+b_{j}\right)
$$

where

$h_{i}$ is the actual output of hidden neuron $\mathrm{j}$.

$P_{i}$ is the input signal of input neuron $\mathrm{i}$.

$W_{i j}$ is the weight between input neuron $\mathrm{i}$ and hidden neuron $\mathrm{j}$.

$b_{j}$ is the bias of hidden neuron $\mathrm{j}$.

$f$ is the activition function.

3. Sums weighted output of hidden layer and apply activition function to compute the output of output layer neurons using:

$$
a_{k}=f\left(\sum_{k=1}^{n} h_{i} W_{i k}+b_{k}\right)
$$

where

$a_{k}$ is the actual output of output neuron $\mathrm{k}$.

$W_{i j}$ is the weight between hidden neuron $\mathrm{j}$ and output neuron $\mathrm{k}$.

$b_{k}$ is the bias of the output neuron $\mathrm{k}$.

4. Compute backpropagation error using:

$$
\delta_{k}=\left(t_{k}-a_{k}\right) f^{\prime}\left(\sum_{k=1}^{n} h_{i} W_{j k}+b_{k}\right)
$$

where

$f^{\prime}$ is the derivative of the activation function. 
$t_{k}$ is the target output neuron $\mathrm{k}$.

5. Calculate weight and bias correction output layer using:

$$
\begin{gathered}
\Delta W_{j k}(n)=\alpha \delta_{k} h_{j} \\
\Delta b_{k}(n)=\alpha \delta_{k}
\end{gathered}
$$

where

$\alpha$ is the learning rate.

6. Add delta input for each hidden unit and calculate the error term using:

$$
\delta_{j}=\sum_{j=1}^{n} \delta_{k} W_{j k} f^{\prime}\left(\sum_{j=1}^{n} P_{i} W_{i j}+b_{j}\right)
$$

7. Calculate the weight and the bais correction for the hidden layer using:

$$
\begin{gathered}
\Delta W i j(n)=\alpha \delta_{j} P_{i} \\
\Delta b_{j}(n)=\alpha \delta_{j}
\end{gathered}
$$

8. Update weights and biases using:

$$
\begin{aligned}
& W_{j k}(n)=W_{j k}(n-1)+\Delta W_{j k}(n) \\
& b_{k}(n)=b_{k}(n-1)+\Delta b_{k}(n) \\
& W_{i j}(n)=W_{i j}(n-1)+\Delta W_{i j}(n) \\
& b_{j}(n)=b_{j}(n-1)+\Delta b_{j}(n)
\end{aligned}
$$

9.Repeat from step 2 to 8 if error tolerance is not satisfied.

The randomness of the generated key from the backpropagation neural network can be justified using statistical tests. If the justification passees, the key can be used for data encryption.

\section{Computer Simulation and Evalution of the PN Sequence Generator}

In this section some of the statistical randomness tests have been applied to binary sequences to test these sequences. These tests include frequency, poker, serial and autocorrelation tests.

\section{- The Frequency Test}

The frequency test involves the calculation of $\chi^{2}$ using[2]:

$$
\chi^{2}=\left(N_{0}-N_{1}\right)^{2} / N
$$

where

$N_{0}$ is the number of occurrences of 0 's in the N-bit sequence.

$N_{1}$ is the number of occurrences of 1's in the N-bit sequence.

The results showing the value of $\chi^{2}$ for different values of $N$ is as shown in Table(1).

Table (1): Frequency test result for the different length sequence.

\begin{tabular}{|c|c|}
\hline Size of file & Result \\
\hline 100 bits & 0.112 \\
\hline 200 bits & 0.154 \\
\hline 300 bits & 0.193 \\
\hline 400 bits & 0.211 \\
\hline 500 bits & 0.285 \\
\hline
\end{tabular}


Here, if $\chi^{2}$ is less than or equal 0.384 then the sequence is said to pass this test, otherwise it is failed. the results presented in Table(1) clearly shows that the proposed sequence passes the frequency test.

\section{- The Serial Test}

The serial test involves the calculation of $\chi^{2}$ using[2]:

$$
\chi^{2}=\left[4 /(N-1) \sum_{i=0}^{1} \sum_{j=0}^{1} N_{i j}^{2}-(2 / N)\left(\sum_{i=0}^{1} N_{i}^{2}\right)\right]+1
$$

where $N_{00}, N_{01}, N_{10}$ and $N_{11}$ be the number of occurrences of $00,01,10$ and 11 respectively in the $\mathrm{N}$-bit sequence. The results showing the value of $\chi^{2}$ for different value of $\mathrm{N}$ as shown Table (2). Here, if $\chi^{2}$ is less than or equal 5.99 then the sequence is said to pass this test, otherwise it is failed. The results presented in Table (2) clearly show that the proposed sequence passes the serial test.

Table (2): Serial test result for the different length sequences.

\begin{tabular}{|c|c|}
\hline Size of file & Result \\
\hline 100 bits & 2.91 \\
\hline 200 bits & 3.45 \\
\hline 300 bits & 3.86 \\
\hline 400 bits & 4.17 \\
\hline 500 bits & 4.43 \\
\hline
\end{tabular}

\section{-The Poker Test}

The poker test involves the calculation of $\chi^{2}$ using[2]:

$$
\chi 2=\left(2^{m} / K\right) \sum_{i=0}^{2^{m}-1}\left(Y_{i}^{2} / C^{m}{ }_{i}\right)-K .
$$

where

$\mathrm{K}$ is the number of blocks.

$\mathrm{M}$ is the length of block

$Y_{i}$ is the number of m-bit subsequences having i 1's and (m-i) 0 's, and so on. The calculated value should be compared table for $\chi^{2}$ with $2^{m}-1$ degrees of freedom. This will find the critical region as before using a statistical table. This test can be applied as many times for different values of $\mathrm{m}$. The results showing the value of $\chi^{2}$ for different value of $m$ is as shown Table(3) for different degrees of freedom.

Table (3): Poker test result for the different length sequences and different degree of freedom.

\begin{tabular}{|c|c|c|c|}
\hline Size of file & $\mathrm{m}=3$ & $\mathrm{~m}=4$ & $\mathrm{~m}=5$ \\
\hline 100 bits & 1.7 & 5.3 & 6.7 \\
\hline 200 bits & 2.0 & 6.7 & 7.2 \\
\hline 300 bits & 2.5 & 7.3 & 8.6 \\
\hline 400 bits & 2.7 & 7.9 & 9.1 \\
\hline 500 bits & 3.1 & 8.4 & 10.3 \\
\hline
\end{tabular}


Here, if $\chi^{2}$ is less than or equal 11.1 then the sequence is said to pass this test, otherwise it is failed. the results presented in Table(3) clearly show that the proposed sequence passes the poker test.

\section{-The Autocorrelation Test}

The autocorrelation test equation involves the calculation of $\chi^{2}$ using :

$$
\mathrm{A}(\mathrm{d})=\sum_{i=1}^{N-d} U_{i} \oplus U_{i+d}
$$

Where $\mathrm{N}$-bit is length of the sequence is $(\mathrm{U})=U_{1} U_{2} \ldots U_{N}$. The factor of $\mathrm{d}$ is shift in bits between the sequences $U_{i}$ and $U_{i+d}$. If there is correlation between the sequences, $\mathrm{A}(\mathrm{d})$ should satisfy on the following relations[2]:

$$
A(d)>((N-d) / 2)+1.96(\sqrt{(N-d)} / 2)
$$

Or

$$
A(d)<((N-d) / 2)-1.96(\sqrt{(N-d)} / 2)
$$

Table (4): Autocorrelation test results for the different length sequences .

\begin{tabular}{|c|c|c|c|c|c|}
\hline No of shift & $\mathrm{N}=100$ & $\mathrm{~N}=200$ & $\mathrm{~N}=300$ & $\mathrm{~N}=400$ & $\mathrm{~N}=500$ \\
\hline 1 & 0.081 & 0.321 & 0.513 & 0.521 & 0.613 \\
\hline 2 & 0.123 & 0.412 & 0.312 & 0.151 & 0.437 \\
\hline 3 & 0.032 & 1.212 & 0.784 & 0.763 & 1.617 \\
\hline 4 & 0.534 & 0.785 & 1.812 & 1.561 & 0.571 \\
\hline 5 & 0.385 & 0.052 & 0.789 & 0.863 & 0.387 \\
\hline 6 & 1.311 & 0.612 & 0.421 & 0.313 & 1.815 \\
\hline 7 & 0.423 & 0.423 & 0.672 & 1.253 & 0.649 \\
\hline 8 & 0.678 & 0.678 & 0.516 & 0.651 & 0.745 \\
\hline 9 & 0.745 & 0.745 & 0.858 & 0.451 & 0.517 \\
\hline 10 & 0.856 & 0.856 & 1.812 & 0.713 & 0.887 \\
\hline
\end{tabular}

It is clear from Table (4) that all sequences tested pass the autocorrelation test since the value of $\mathrm{A}(\mathrm{d})$ for all are less than 1.96 as recommended by equation 21 .

\section{The Proposed Artificial Neural Network Scrambling Function}

A new approach for data scrambling has been proposed from the standard backpropagation algorithm. The proposed approach uses the generated key from the neural network proposed in section 4 as input with the key weight instead of bias and plaintext as the second input as shown by the flowchart shown in Figure (3). The same technique is used for decrypting the ciphertext shown by the flowchart shown in Figure (4). The procedure used in the proposed algorithm is as follow:

1.Initiliazation network weight values

2.Sums weighted input and apply activition function to compute the output of the hidden layer using:

$$
h_{i}=f\left(\sum_{i=1}^{n} P_{i} W_{i j}+\sum_{i=1}^{n} K i W k_{i j}\right)
$$

where 
$h_{i}$ is the the actual output of hidden neuron $\mathrm{j}$.

$P_{i}$ is the input signal of input neuron i.

$W_{i j}$ is the weight between input neuron $\mathrm{i}$ and hidden neuron $\mathrm{j}$.

$K_{i}$ is the key of hidden neuron $\mathrm{j}$.

$W k_{i j}$ is the key weight.

$f$ is the the activition function.

3. Sums weighted output of hidden layer and apply activition function to compute the output of output layer neurons using:

$$
a_{k}=f\left(\sum_{k=1}^{n} h_{i} W_{i k}+\sum_{k=1}^{n} K_{i} W k_{i k}\right)
$$

where

$a_{k}$ is the the actual output of output neuron $\mathrm{k}$.

$W_{i j}$ is the weight between hidden neuron $\mathrm{j}$ and output neuron $\mathrm{k}$.

4. Compute backpropagation error using:

$$
\delta_{k}=\left(t_{k}-a_{k}\right) f^{\prime}\left(\sum_{k=1}^{n} h_{i} W_{j k}+\sum_{k=1}^{n} K_{i} W k_{j k}\right.
$$

where

$f^{\prime}$ is the the derivative of the activation function.

$t_{k}$ is the the target output neuron $\mathrm{k}$.

5. Calculate weight and key weight correction output layer using:

$$
\begin{aligned}
& \Delta W_{j k}(n)=\alpha \delta_{k} h_{j} \\
& \Delta W k_{j k}(n)=\alpha \delta_{k}
\end{aligned}
$$

where

$\alpha$ is the learning rate.

6. Add delta input for each hidden unit and calculate the error term using:

$$
\delta_{j}=\sum_{j=1}^{n} \delta_{k} W_{j k} f^{\prime}\left(\sum_{j=1}^{n} P_{i} W_{i j}+\sum_{j=1}^{n} K_{i} W k_{i j}\right)
$$

7. Calculate the weight and the key weight correction for the hidden layer.

$$
\begin{aligned}
& \Delta W i j(n)=\alpha \delta_{j} P_{i} \\
& \Delta W k_{i j}(n)=\alpha \delta_{j}
\end{aligned}
$$

8. Update weights and biases.

$$
\begin{aligned}
& W_{j k}(n)=W_{j k}(n-1)+\Delta W_{j k}(n) \\
& W k_{j k}(n)=W k_{j k}(n-1)+\Delta W k_{j k}(n) \\
& W_{i j}(n)=W_{i j}(n-1)+\Delta W_{i j}(n) \\
& W k_{i j}(n)=W k_{i j}(n-1)+\Delta W k_{i j}(n)
\end{aligned}
$$

9.Repeat from step 2 to 8 if error tolerance is not satisfied. 


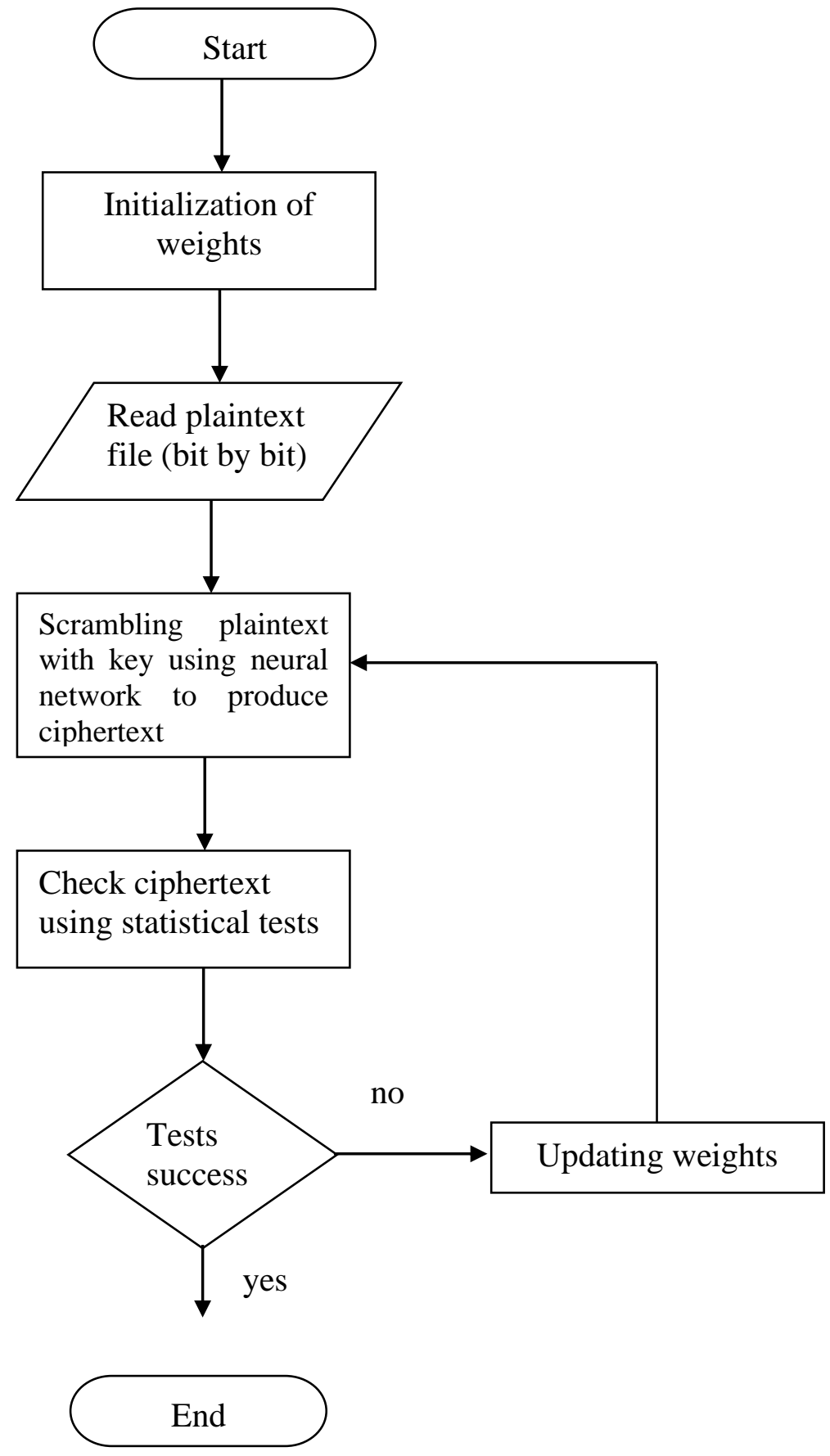

Figure.(3): Flowchat of proposed system for data encryption. 


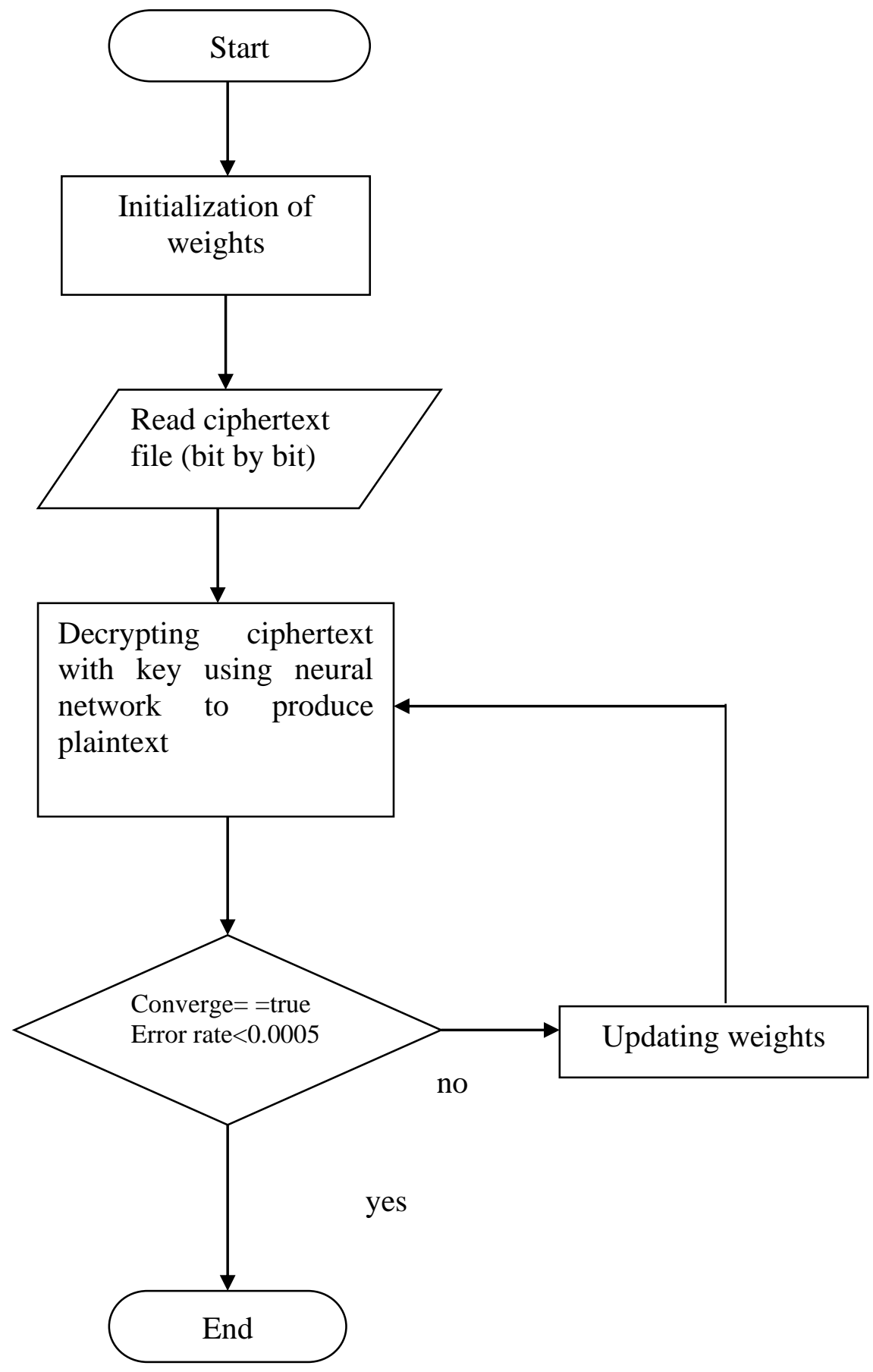

Figure.(4): Flowchart of proposed system for data decryption.

The proposed scrambling technique has been evaluated by encrypting the following plaintext with the generating key (Section 4). The following plaintext is adopted as input

There are several network topologies. Each topology is designed to solve a specific problem or to represent certain point of view. Each topology has some drawbacks and benefits. These topologies can be categorized in three main categories: Fully interconnected, feed forward connection, and mixed connectivity. 
With the following key

1010100010100000000000001100010110100100000001001000001000000000000000100100001100000 1001000100001000000000000010001001000100000010001000000001000100101001100000000010001 0100010000000111011001001000000001000101001010011100101001010000001101000000000001000 0000100010001011100001000000000001101001100000000000000010000001000000100101101000010 0000010000110101011000010000010000010000100000001001100000000110000101110000010010000 0010000100001010100000001000000000010000010100001000011000001000100101011100000010110 0000000010000100010101001101111110000001010010000000010000111100000000000101000110100 0111000001000100000011101001010000000000001010000010100010000000001000000110001101000 0101100000010000010100100001100001001000010001001000000101010110000000010000000111000 00100010110000000000000000010000000010000001000000001000000000100001010000000000001010 1100000010000000101000001000000000000000100001000100000000000100000001000001000100110 0001010001100101000001100010000100010000110000001000000001101100100010100000010100000 0000010000010001001100000100101000000100010101000100000110000001000000110110001010010 0000000100101001000101110000000001000001000010000000001111000100000001110001011101000 00100011000100100010001010010000000010010000000000000100000000000000010100011001000111 0110000000000000001001011010000001101000010010100110000100001110000010001000000000000 1100000000001000000010100110000000000100000100110011000110000010000000000000010110000 0011101000000100110011000000111011001101000000000100110000101101000000000000000000101 1110100110010100100100110000000001001000001010000000001010000100000000001101001000110 0010100000010000001010100011111001000010010001000000000000001000101000000001000000010 0000000101110010001000010010010000000001000100110010000001001000001111000000000000100 0000010100000000000000001011001000101000011000000010000000000000011100000001000110000 1000110100000001110000100000000000010000001000100000001100000001100101011000001000000 0000001000000001000000000000101100101100001010000001100001011100000001001001000000000 1010000111000100000000000000000000110001000010011100010010000010000000011000000100000 0100000000101010011

This will produce the following ciphertext

4harm'oR qe|\%n“QQ k3ض33\% dO|nl\{\#ée)*TJihf?/ovë\&[\$iâ( ;p'zdS)dت1/4! mfu@

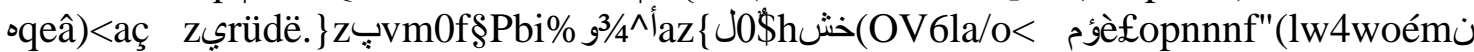

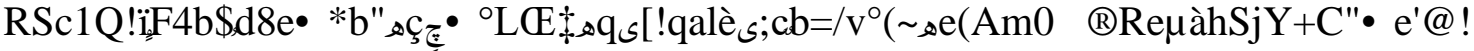
ûdW ut|"|نmr'nll APd.•etpfiT\}bpd'§ eVa4;mc6\$Jne€AH \$2\#;fne@s!fTi;

\section{Conclusions}

There are several techniques that can be used to generate a PN sequence. However, a new cryptosystem based on neural network is found very attractive in the field cryptosystem. Neural network technique is used for key generation and to encrypt data. The proposed technique offers more randomness for key generation, also to reduce the process time and to get high reliable secure system as compare as the traditional stream cipher methods.

\section{REFERENCES}


[1] M. Prokop, 2004, " Stream cipher", http//www.michal-prokop.at.

[2] A. Kanso, 1999, "Clock-controlled generators", Ph.D. thesis, University of London.

[3] P. Inakollu, 2003, "A study of the effectiveness of neural network for elemental concentration from libs spectra", MS.C. thesis, Mississippi state university.

[4] A. Klimov, A. Milyaguine, and A. Shamir,2002,"Analysis of neural network", Computer science department, http://citeseer.ist.psu.edu/kinzel02neural.html.

[5] A. Albassal and Dr.A.Wahdan,2004,"A neural network based block cipher", Computer and system engineering Dept. faculty of engineering, Ain shams university.

[6] P. Ekdahl, 2003, "On LFSR based stream ciphers analysis and design",Ph.D. thesis, Lund university.

[7] J. Buchmann, 2001, "Introduction to cryptography", Edwards brothers publishing.

[8] T. Lindquist, M. Diarra, and B. Millard, 2004, ”A java cryptography service provider implementing one-time pad", Proceedings of the 37th hawaii international conference on system sciences.

[9] C. Tegiou and D.Sigano,"Neural network", 2000, http://www.doc.ic.ac.uk/ nd/ surprise96.

[10] M. Hagan, H. Demuth and M. Beale, 1996, "Neural network design”, A division of international Thomson publishing Inc.

[11] R. Callan, 1999, “The essence of neural networkg”, Prentice hall europ.

[12] M. Manic. 2008, "Neural networks design", http://husky.if.uidaho.edu/ ee578s08/ hw.html 